\title{
Precordial Heart Image Disappearance: An Uncommon New Specific Ultrasound Sign for Diagnosing Neonatal Pneumothorax and its Thoracentesis Treatment at the Precardiac Area- an Pilot Study Reports from Two Centers
}

Jing Liu ( $\square$ liujingbj@live.cn )

Department of Neonatology \& NICU,Beijing Chaoyang District Maternal and Child Healthcare Hospital https://orcid.org/0000-0002-1735-840X

\section{Wei Yan}

Department of Ultrasound,Henan Zhumadian Center Hospital

Jian-Wei Yang

Department of Ultrasound,Henan Zhumadian Center Hospital

Guo Guo

Department of Neonatology \& NICU,Beijing Chaoyang District Maternal and Child Healthcare Hospital Ru-Xin Qiu

Department of Neonatology and NICU,Beijing Chaoyang District Maternal and Child Healthcare Hospital

\section{Research}

Keywords: lung ultrasonography,pneumothorax,lung disease,newborn infant,precordial heart image,x-ray

Posted Date: August 23rd, 2021

DOI: https://doi.org/10.21203/rs.3.rs-808576/v1

License: (c) (i) This work is licensed under a Creative Commons Attribution 4.0 International License. Read Full License 


\section{Abstract}

Background: Use of lung ultrasonography (LUS) to diagnose neonatal pneumothorax (PTX) has been well documented. This study aimed to emphasize the value of precordial heart image (PHI) disappearance as an ultrasound sign to diagnose neonatal PTX and to perform thoracentesis at the precardiac area in patients with PTX.

Methods: From March 2017 to May 2021,a total of 288 infants were included in this retrospective study, including 48 patients with PTX,48 patients with respiratory distress syndrome,48 patients with transient tachypnea,48 patients with meconium aspiration syndrome,48 patients with pneumonia,and 48 patients with normal lung tissue.LUS was performed routinely,and thoracentesis was performed under LUS guidance.

Results: Eight of 48 patients with PTX (16.7\%) presented with PHI disappearance on LUS,which exhibited a sensitivity of $16.7 \%$ and a specificity of $100 \%$ for diagnosing neonatal PTX.Needle aspiration was performed on 42 patients with PTX (87.5\%),8 of whom underwent puncture at the precardiac area. Satisfying results were obtained in 40 patients $(95.2 \%)$ without any complications.

Conclusion: PHI disappearance is an uncommon ultrasound sign on LUS that can be used to diagnose neonatal PTX.Selection of the precardiac area as the puncture site is safe for the treatment of neonatal PTX in patients with PHI disappearance.

\section{Introduction}

Pneumothorax (PTX) occurs when gas accumulates in the pleural space.PTX is one of the common complications of neonatal pulmonary disease,and it leads to a significant increase in neonatal mortality.A retrospective survey from Canada on 71,237 newborn infants indicated that the neonatal population incidence rate of PTX was $4.0 \%$ in early preterm (gestational age $[\mathrm{GA}]<32$ weeks), $2.6 \%$ in moderate-late preterm (GA 32-36 weeks),and 6.7\% in term (GA $\geq 37$ weeks) neonates [1].Another investigation from the US showed that in 13,811 newborn infants,the prevalence of PTX was $0.27 \%$ in infants with a birth weight of $<2500 \mathrm{~g}$ and $2.5 \%$ in infants with a birth weight of $\geq 2500 \mathrm{~g}$ [2].PTX is not only associated with a 30\% increase in mortality with an odds ratio of 5.27 (95\% confidence interval $1.96-14.17)[3,4]$, but it is also associated with a high rate of bronchopulmonary dysplasia,severe intraventricular hemorrhage,and a prolonged neonatal intensive care unit (NICU) length of stay,especially in early preterm neonates [1-4].Therefore,early and accurate diagnosis followed by correct treatment is imperative to improve the prognosis of patients with PTX.

PTX is traditionally diagnosed by chest X-ray or computed tomography (CT),both in adults and children [5-7]. However,there is a certain missed diagnosis rate in radiological diagnosis,especially for occult PTX [8].Moreover,there is inevitable radiation damage associated with CT.Radiation damage is particularly severe for growing newborns and premature babies.Recently,lung ultrasonography (LUS) has been successfully used to diagnose PTX.Clinical studies and literatures have proved that the sensitivity and 
specificity of LUS in the diagnosis of PTX,both in adults and children, are superior to X-ray [9-11]. Relevant guidelines have been published to promote the application of LUS in the diagnosis and treatment of neonatal lung diseases and PTX [12-15].

Generally,LUS is used to diagnose PTX based on the following ultrasound characteristics $[13,14]:(1)$ disappearance of lung sliding;(2) absence of B lines;(3) presence of pleural lines and A lines;(4) sandy beach signs replaced by stratosphere signs on M-mode imaging;(5) presence of lung point in mild-tomoderate PTX.

Recently,we identified a new LUS sign that could be used to diagnose neonatal PTX,that is,precordial heart image (PHI) disappearance.To promote PTX diagnosis using LUS,this new sign is introduced in this paper.

\section{Materials And Methods \\ Study setting}

The study was conducted at the NICUs of two tertiary hospitals.

\section{Ethics statement}

The study protocol was approved by the ethics committees of Beijing Chaoyang District Maternal and Child Healthcare Hospital and Henan Zhumadian City Center Hospital. All methods were performed in accordance with the relevant guidelines and regulations. Written informed consent was obtained from participants' parents.

\section{Instruments and examination methods}

A Voluson S10 or Voluson E 10 (GE Healthcare, USA) ultrasound system with a linear array probe (frequency $>10 \mathrm{MHz}$ ) was used for LUS examination. All examinations were strictly performed in accordance with published guidelines and specifications [11, 12].PTX was diagnosed according to criteria for LUS diagnosis of PTX $[13,14]$.

\section{Participants}

From March 2017 to May 2021,a total of 48 patients with PTX were diagnosed at the NICUs of Beijing Chaoyang District Maternal and Child Healthcare Hospital and Henan Zhumadian City Center Hospital.In a ratio of 1:1,48 neonates with no lung disease,48 patients with respiratory distress syndrome (RDS),48 patients with transient tachypnea (TTN),48 patients with meconium aspiration syndrome (MAS), and 48 patients with pneumonia were randomly selected as controls.Therefore,a total of 288 infants were included in this retrospective study. All LUS examinations were performed by well-trained neonatologists.Clinical data were collected by different doctors, and the LUS operator was blinded to the clinical conditions of neonates. 


\section{Observation indices}

PHI was the key observation. Traditional signs,such as pleural lines,A lines, B lines,lung consolidation,lung sliding,lung point,and the stratosphere sign,were also examined [12-14].

\section{Thoracentesis at the precardiac area}

In PTX patients with a PHI,thoracentesis was performed according to internal guidelines [13]. In PTX patients with PHI disappearance,thoracentesis was performed at the precardiac area under LUS monitoring.

\section{Statistical analysis}

SPSS 21.0 software was used to conduct the statistical analysis.Fisher's exact test was used to compare the rate of positive neonatal LUS findings between groups.The specificity and sensitivity of PHI disappearance for diagnosing PTX were calculated based on Fisher's exact test. A $p$ value of $<0.05$ was considered statistically significant.

\section{Results}

\section{Clinical and demographic data}

The GA of this study population ranged from $27 \pm 3$ weeks to $41 \pm 1$ week. The study population consisted of 99 preterm infants and 189 full-term infants. The birth weight ranged from 880-4210 g.There were 156 males and 132 females. A total of 176 infants were delivered by vaginal delivery,while 112 infants were delivered by cesarean section.

The study subjects included 48 patients with PTX and 240 controls. The control subjects included 48 infants with healthy lungs,and 192 infants with different types of lung disease (48 cases of RDS,48 cases of TTN,48 cases of MAS, and 48 cases of pneumonia). There were no significant differences in GA and birth weight among the subgroups.

\section{Normal LUS manifestations}

Healthy neonatal lung demonstrates a bamboo sign on B-mode LUS (Fig. 1A) and a seashore sign (Fig. 1B) on M-mode LUS.Lung sliding is clearly evident on real-time LUS (Video 1) [12, 13].In addition,the heart image in the left precardiac area is visible in healthy newborns.Moreover,heart movement should be visible on real-time LUS (Video 2).

\section{Ultrasound manifestations of PTX}

The major LUS manifestations of PTX are summarized in Table 1.These include (1) disappearance of lung sliding on real-time LUS (in this study,disappearance of lung sliding was observed in all patients with 
PTX);(2) absence of B lines (all patients lacked B lines at PTX sites);(3) presence of pleural and A lines (all patients demonstrated pleural and A lines at PTX sites);(4) lung point (which was observed in 30 cases [62.5\%] of mild and moderate PTX);(5) spared areas (which were observed in 12 patients with PTX [35\%]);(6) the stratosphere sign (which was seen in all patients with PTX at the PTX site);and (7) PHI disappearance (8 patients with PTX [16.7\%] demonstrated PHI disappearance).

\section{Sensitivity and specificity of PHI disappearance in PTX diagnosis}

Many LUS signs,such as presence of pleural and A lines,absence of B lines,lung sliding disappearance, lung point in mild-to-moderate PTX,spared areas in patients with mild PTX,and the stratosphere sign on M-mode LUS, are used to diagnose PTX [10-14, 16, 17].However,PHI disappearance has not been described previously. Thus, we investigated the value of $\mathrm{PHI}$ disappearance for the diagnosis of neonatal PTX using LUS in this study. Specifically,PHI disappearance was used as a parameter to calculate the sensitivity and specificity of LUS to diagnose neonatal PTX.The results indicate that PHI disappearance exhibits a sensitivity of $16.7 \%$ and a specificity of $100 \%$ for diagnosing neonatal PTX (Table 2 ).

Effectiveness of thoracentesis

Among the 48 patients with PTX,needle aspiration was performed in 42 patients (87.5\%) with significant clinical symptoms under LUS monitoring.Satisfying results were obtained in 40 patients $(95.2 \%)$,and gas was completely removed after puncture. The remaining 2 cases underwent closed thoracic drainage for $1-3$ days. The drainage gas volume was $55-520 \mathrm{~mL}$.

The puncture site was at the second intercostal space of the left or right midclavicular line (the traditional puncture site) in 14 cases;at the fifth to sixth intercostal space of the left or right midaxillary line in 12 cases;at the seventh to eighth intercostal space of the left or right subscapular line in 8 cases;and at the precardiac area in 8 cases,including 5 cases at the sixth intercostal space of the left midclavicular line and 3 cases $1 \mathrm{~cm}$ medial to the fifth intercostal space of the left midclavicular line.

Typical case presentation

To aid understanding of the results,we herein present a typical case.An infant with a GA of $32 \pm 1$ week,who was delivered by caesarean section with a birth weight of $1980 \mathrm{~g}$,was admitted to the NICU because of severe dyspnea 3 hours after birth.LUS confirmed RDS in the right lung.Pleural lines and A lines were observed in the regions of L1-L2, L3-L4, and L5-L6, but no heart image or heart movement was observed (Video 3).Lung sliding also disappeared (Videos 4 \& 5).

When $75 \mathrm{~mL}$ of gas was removed by pleural puncture from the sixth intercostal space at the left midclavicular line (Fig. 2), dyspnea was relieved,and heart rate decreased from more than $180 \sim 200$ beats/min to approximately 140 beats/min.LUS showed that the heart image and heart movement emerged in the left precardiac region (L1-L2) (Video 6) after thoracic puncture.Therefore,it was confirmed that PHI disappearance was caused by left-sided PTX. 


\section{Discussion}

Use of LUS to diagnose neonatal PTX has been documented,and this approach has a higher sensitivity than traditional chest X-ray [11-14, 16-20].The major LUS signs of PTX are disappearance of lung sliding, absence of $B$ lines,presence of pleural and A lines,and/or presence of lung point [11-14, 1620].This study is the first to report that PHI image disappearance is an unusual but specific LUS sign that can be used to diagnose neonatal PTX.

In the present study,PHI disappearance was only observed in $16.7 \%$ of patients with PTX, while it was not observed in patients with TTN,RDS,MAS,pneumonia,or healthy lungs. Thus, we believe that PHI disappearance is an uncommon,yet specific,LUS sign of neonatal PTX. The sensitivity and specificity of PHI disappearance were $16.7 \%$ and $100 \%$, respectively.

Previously,the most commonly selected puncture site for the treatment of PTX based on chest X-ray diagnosis was the second intercostal space in the midclavicular line.However, thoracic puncture under LUS completely changed this opinion.According to our experience and international expert recommendations,suitable puncture sites are generally as follows [13]: (1) the intercostal space where pleural and A lines exist in B-mode;(2) the intercostal space where lung sliding disappears on real-time LUS; and (3) the intercostal space that presents with a stratosphere sign in M-mode.However,according to the results of this study,for patients with $\mathrm{PHI}$ disappearance,we can also select the precardiac area as the puncture site.Eight patients underwent puncture at this area, including 5 cases at the sixth intercostal space of the left midclavicular line and 3 cases $1 \mathrm{~cm}$ medial to the fifth intercostal space of the left midclavicular line.To our knowledge,this is the first report to use the precardiac area as the puncture site for the treatment of neonatal PTX.Performing pleural puncture in this area to treat PTX would not have been possible before LUS was developed.

\section{Limitations}

This study has some limitations that should be noted.First,LUS examinations were performed by welltrained doctors; thus, the results may not apply in the hands of less experienced examiners.Second,we did not measure inter-observer or intra-observer variability.Any physician who wishes to perform LUS should be properly trained $[21,22]$.Third,the sample size of this study was small,so there may be some deviation in the results. Therefore,further expansion of the sample size and prospective multi-center studies are needed to verify the observations.

\section{Conclusion}

In conclusion,the present study performed an in-depth investigation of the LUS characteristics of neonatal PTX and its treatment under LUS monitoring.It was confirmed that PHI disappearance is an uncommon and specific sign that could be used to diagnose neonatal PTX.Moreover,use of the 
precardiac area as the puncture site was safe for the treatment of neonatal PTX in patients with PHI disappearance in this study.

\section{Abbreviations}

PTX

Pneumothorax; RDS = Respiratory distress syndrome;LUS = Lung ultrasound;NICU = Neonatal intensive care unit;TTN = Transient tachypnea of the newborn; MAS = Meconium aspiration syndrome; $\mathrm{PHI}=$ Precordial heart image;GA = Gestational age .

\section{Declarations}

\section{Ethical Approval and Consent to participate}

The study protocol was approved by the ethics committees of Beijing Chaoyang District Maternal and Child Healthcare Hospital and Henan Zhumadian City Center Hospital. All methods were performed in accordance with the relevant guidelines and regulations. Written informed consent was obtained from participants' parents.

\section{Authors' contributions}

JLcontributed to the study conception,ultrasound examination, and data analysis,and wrote and approved the manuscript.WYcontributed to clinical data analysis,manuscript preparation,and approval of the final manuscript.JWY contributed to clinical data analysis, manuscript preparation,and approval of the final manuscript.GGcontributed to clinical data collection,manuscript revision, and approval of the final manuscript.RXQcontributed to data collection,manuscript revision,and approval of the final manuscript.

\section{Availability of supporting data}

The data set used and analyzed are available from the corresponding author on reasonable request.

\section{Funding source}

This work was supported by a grant from the Social Development Projects,Beijing Chaoyang District Bureau of Science,Technology and Information (SYSF1820,CYSF1922).

\section{Finlacial disclosures}

The authors have no financial relationships relevant to this article to disclose.

Conflict of Interest Statement: No

Consent for publication 
Not applicable.

\section{Acknowledgments}

We thank Emily Woodhouse,PhD,from Liwen Bianji (Edanz) (www.liwenbianji.cn/),for editing the English text of a draft of this manuscript.

\section{References}

1. Duong HH, Mirea L, Shah PS, Yang J, Lee SK, Sankaran K. Pneumothorax in neonates: Trends, predictors and outcomes. J Neonatal Perinatal Med. 2014;7(1):29-38. doi:10.3233/NPM-1473813.

2. Aly $\mathrm{H}$, Massaro $A$, Acun $\mathrm{C}, \mathrm{Ozen} \mathrm{M}$ Pneumothorax in the newborn: clinical presentation, risk factors and outcomes. The Journal of Maternal- Fetal \& Neonatal Medicine,2014;27(4):402-6. doi: 10.3109/14767058. 2013.818114.

3. Jaroensri S, Kamolvisit W, Nakwan N. Risk factor analysis of pneumothorax associated with persistent pulmonary hypertension of the newborn in Thai neonates. The Journal of Maternal- Fetal Neonatal Medicine,2019;doi:10.1080 /14767058.2019.1596403.

4. Apiliogullari B, Sunam GS, Ceran S, Koc H. Evaluation of neonatal pneumothorax. J Int Med Res. 2011;39(6):2436-40.

5. Musalar E. Ekinci S,Ünek O,Arş Eda,Eren HŞ,Gürses B, Aktaş C.Conventional vs invert-grayscale X-ray for diagnosis of pneumothorax in the emergency setting. Am J Emerg Med. 2017;35(9):1217-21.

6. doi: 10.1016/j.ajem.2017.03.031.

7. Soccorso G, Anbarasan R,Singh M, Lindley RM,Marven SS,Parikh DH.Management of large primary spontaneous pneumothorax in children: radiological guidance,surgical intervention and proposed guideline. Pediatr Surg Int,2015 Dec;31(12):1139-44. doi: 10.1007/s00383-015-3787-8.

8. Erez D, Israeli-Shani L, Shochet GE, King DA, Abu-Akel M. Dovrish Z,Shitrit D.Clinical and Radiological Characteristics of Patients Diagnosed with Spontaneous Pneumothorax:Treatment Options and Clinical Outcomes. A Retrospective Analysis 2004 to 2017. Isr Med Assoc J. 2020;22(12):747-51.

9. Malek D, Santillanes G, Hsiao V, Mailhot T, Claudius IOccult. Pneumothorax Identification on Extended Focused Assessment with Sonography for Trauma Examination in Children. Pediatr Emerg Care,2020,doi: 10.1097/PEC.0000000000002303.Online ahead of print.

10. Liu J, Lovrenski J, Hlaing AY, Kurepa D. Neonatal lung diseases: lung ultrasound or chest x-ray. The Journal of Maternal-Fetal \& Neonatal Medicine,2021; 34(:): 1177-1182. https://doi.org/ 10.1080/14767058. 2019.1623198.

11. Dahmarde H, Parooie F,Salarzaei M. Accuracy of Ultrasound in Diagnosis of Pneumothorax: A Comparison between Neonates and Adults-A Systematic Review and Meta-Analysis. Can Respir J,2019;2019:5271982.doi: 10.1155/2019/5271982.

12. Sandig J, Bührer C, Czernik C.Sandig J, et al. Lung Ultrasound in Neonatology to diagnose a Pneumothorax (part one): Evidence - Time for a New Standard Protocol. Z Geburtshilfe Neonatol. 
2021;225(1):15-8. doi:10.1055/a-1312-7773.

13. Liu J, Copetti R,SorantinE, Lovrenski J, Rodriguez-Fanjul J, Kurepa D, Feng X, Cattaross L, Zhang H, Hwang $M$, et al. Protocol and Guidelines for Point-of-Care Lung Ultrasound in Diagnosing Neonatal Pulmonary Diseases Based on International Expert Consensus. J Vis Exp. 2019;;145:e58990. doi:10.3791/58990.

14. Liu J, Kurepa D, Feletti F, Alonso-Ojembarrena A, Lovrenski J, Copetti R, Sorantin E, Rodriguez-Fanjul $\mathrm{J}$, Katti K, Aliverti A, et al. International Expert Consensus and Recommendations for Neonatal Pneumothorax Ultrasound Diagnosis and Ultrasound-guided Thoracentesis Procedure. J Vis Exp. 2020;157:e60836. doi:10.3791/60836.

15. Sandig J, Bührer C, Czernik C. Lung Ultrasound in Neonatology to diagnose a Pneumothorax (part two): A Guideline. Z Geburtshilfe Neonatol. 2021;225(2):105-10. doi:10.1055/a-1312-7809.

16. Liu J, Guo G, Kurepa D,Volpicelli G, Sorantin E, Lovrenski J, Alonso-Ojembarrena A, Hsieh KS, Lodha A, Yeh TF,et al. Specification and guideline for technical aspects and scanning parameter settings of neonatal lung ultrasound examination. The Journal of Maternal- Fetal \& Neonatal Medicine,2021:114,DOI: $10.1080 / 14767058.2021 .1940943$.

17. Liu J, Chi JH, Ren XL, Li J, Chen YJ, Lu ZL, Liu Y, Fu W, Xia RM.Liu J, et al.Lung ultrasonography to diagnose pneumothorax of the newborn. Am J Emerg Med,2017;35(9):1298-1302.doi:10.1016/ j.ajem. 2017.04.001.

18. Fei Q, Lin Y, Yuan TM.Fei Q, et al. Lung Ultrasound, a Better Choice for Neonatal Pneumothorax: A Systematic Review and Meta-analysis.Ultrasound Med Biol,2021;47(3):359-69.doi:10.1016/ j.ultrasmedbio.

19. Cattarossi L, Copetti R, Brusa G, Pintaldi SLung. Ultrasound Diagnostic Accuracy in Neonatal Pneumothorax. Can Respir J,2016;2016:6515069.doi:10.1155/2016/6515069.

20. Raimondi F, Rodriguez-Fanjul J, Aversa S, Chirico G, Yousef N, De Luca D, Corsini I, Dani C, Grappone L, Orfeo L, Migliaro F. Vallone G,Capasso L,Lung Ultrasound in the Crashing Infant (LUCl) Protocol Study Group. Lung Ultrasound for Diagnosing Pneumothorax in the Critically. III NeonateJ Pediatr. 2016;175(1):74-8.e1. doi:10.1016/j.jpeds.2016.04.018.

21. Deng BY, Li N, Wu WS, He XG,Li JF, Huang TL. Li YC,Jiang SL.Use of Neonatal Lung Ultrasound for the Early Detection of Pneumothorax. Am J Perinatol. 2020;37(9):907-13. doi:10.1055/s-00391688999.

22. Dalibor K, Vitalya B, Nahla Z, et al.Structured neonatal point-of-care ultrasound training program. Am J Perinatol.2020:1-8. DOI:10.1055/s-0040-1709667.

23. Nguyen J, Amirnovin R, Ramanathan R,et al. The state of point-of-care ultrasonography use and training in neonatal-perinatal medicine and pediatric critical care medicine fellowship programs. $J$ Perinatol. 2016;36(11):972-6.

\section{Tables}


Due to technical limitations, table 1 and 2 is only available as a download in the Supplemental Files section.

\section{Figures}

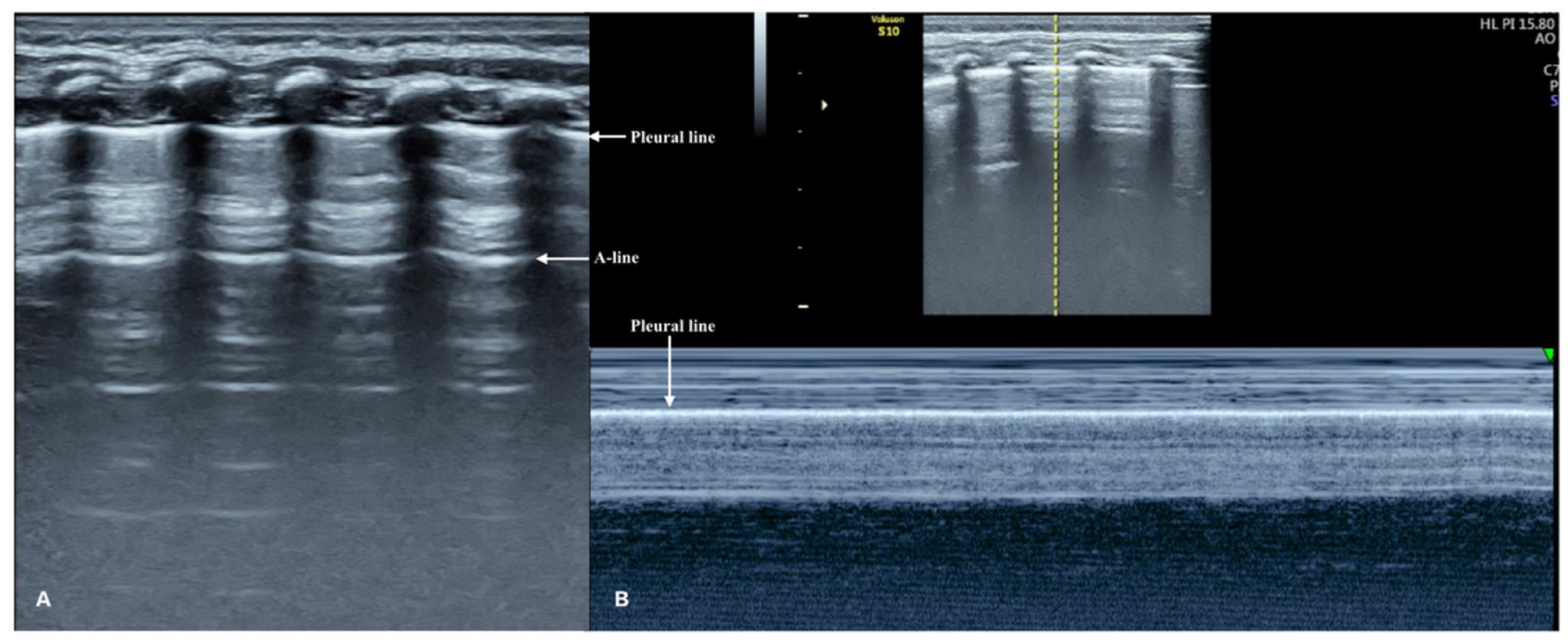

\section{Figure 1}

Healthy lung ultrasound manifestations. The healthy lung presents a bamboo sign on B-mode ultrasound (A) and a seashore sign (B) on M-mode ultrasound, as well as lung sliding (Video 1). The heart image can be seen in the left precardiac area (Video 2) on real-time ultrasound. 


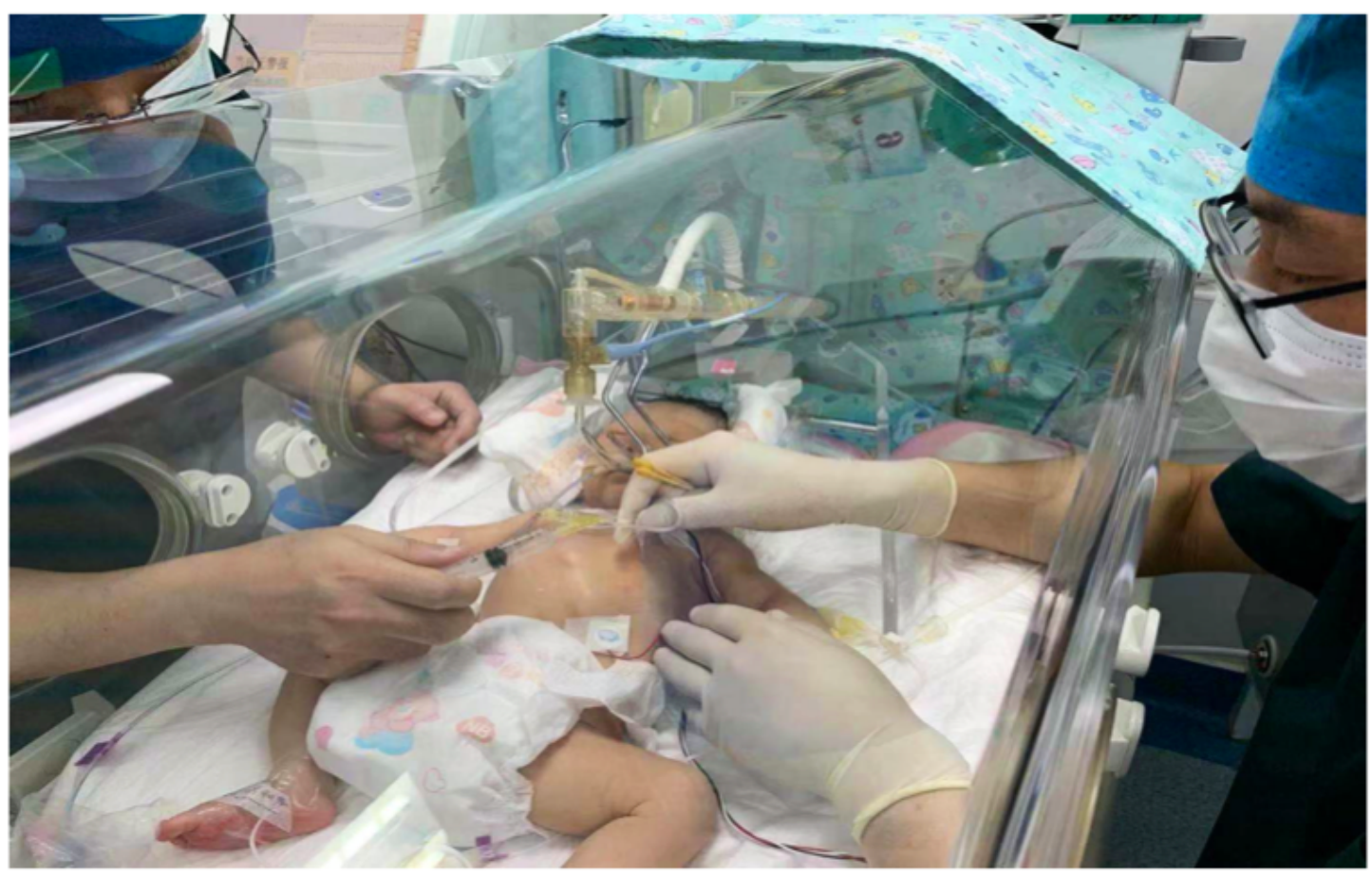

\section{Figure 2}

Pleural puncture. Thoracentesis was performed at the site of the sixth intercostal space at the left midclavicular line. Dyspnea was relieved, and heart rate decreased from more than 180 200 beats/min to approximately 140 beats/min after $75 \mathrm{~mL}$ of gas was removed.

\section{Supplementary Files}

This is a list of supplementary files associated with this preprint. Click to download.

- Tablepages.pdf

- EquatorChecklist.docx

- 12Video1lungsliding.mp4

- Videro2L12PHIdisappearance.mp4

- Videro3L12PHIdisappearance.mp4 
- Video4L34IMG2020061514.mp4

- Video5L56.mp4

- Video6.mov 